\title{
Introduction to Minitrack on Smart City Digital Twins
}

\author{
John E. Taylor \\ Georgia Institute of Technology \\ jet@gatech.edu
}

\author{
Gisele Bennett \\ Florida Institute of Technology \\ gbennett@,fit.edu
}

\author{
Neda Mohammadi \\ Georgia Institute of Technology \\ nedam@gatech.edu
}

Smart City Digital Twins are digital replicas of the city that are progressively informed by the realtime spatiotemporal data from human and infrastructure systems in the real city. These IoTenabled, data-rich virtual platforms, are used to progressively inform decision makers by dynamically simulating changes happening in the city, through integration of cyber-physical city infrastructures that are embedded with information about the dynamics of infrastructure processes, environmental qualities, and human activities, as well as adaptation to changing conditions, to improve cities' resilience, sustainability, and livability [1].

In this Minitrack, we establish the theoretical and scholarly foundation of a Smart City Digital Twin paradigm that enables increased visibility into cities' human-infrastructure-technology interactions involving policy elements through learning, analytics and exchange of spatiotemporal data with particular emphasis (but not limited) to the following topics:

- Theory, Scale, and System Architecture for Smart City Digital Twins.

- Data, Sensing, IoT, and Analytics for Smart City Digital Twins.

- Human-Infrastructure Interdependencies, Connectivity, and Citizen Engagement.

- Information Management and Decision Support for Smart City Digital Twins.

- Digital Twin Virtualization (Virtual Reality / Augmented Reality / Mixed Reality) of Smart Cities.

- Implications for Operational Readiness, ContextAware Simulation, and Crisis Management.

Emerging interdependencies between humans, infrastructures, and technologies as cities implement socio-technical advancements to transform into smarter cities may result in increasing uncertainties, unreliable predictions, and poor management decisions. Decision makers in cities thus require an enhanced ability to model, understand, and anticipate the operational dynamics across human, infrastructure, and technology systems collectively as an integrated entity. However, we lack insights into system behavior differences and scalable data integration approaches at and across scales in relation to city infrastructure systems. Although sensors provide data, sensor data only gives us an abstract view of operations, at best. Nuances of human experience and culture are concealed beyond the sensors' monitoring reach. Understanding these complexities and the dependencies on policy and regulations requires a paradigm shift in how city dynamics are understood, influenced, and ultimately managed. Dynamically enabling simulation of whatif scenarios, Smart City Digital Twins can help analysts and decision makers perceive interdependencies, anticipate emergent behavior, understand how cities equipped with smart technologies will likely perform under various economic, environmental, and social conditions, and identify the drivers of possible disruptions. Such understanding is critical in assessing whether or not smart growth strategies are effective, minimizing the gap between smart utopia and smart reality.

Smart City Digital Twins are complex and require new technological and methodological advancements and collaborative participation across disciplines. Four exemplar papers, aimed at addressing practical and theoretical aspects of such developments, are featured in this edition of the minitrack at HICSS-53. The focus of these papers range from sensor-based analyses and data fusion of human-infrastructure interactions in cities, capturing needs and preferences of urban communities; to assessing city infrastructure resilience, incorporating human response elements such as human sentiment in relation to infrastructure disruptions; to unraveling the complexities of realtime knowledge discovery from heterogeneous city data with Smart City Digital Twins.

\section{References}

[1] Mohammadi N, Taylor JE. Smart City Digital Twins. IEEE Symp. Ser. Comput. Intell., 2017. doi:10.1109/SSCI.2017.8285439. 